Electronic Supplementary Information

Oxidative capacity of nanobubbles and its effect on seed germination

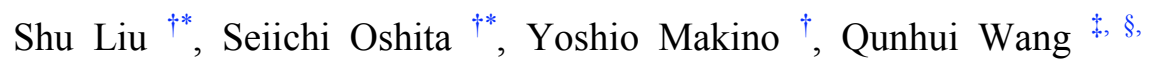
Yoshinori Kawagoe ", Tsutomu Uchida ${ }^{\perp}$

${ }^{\dagger}$ Graduate School of Agricultural \& Life Sciences, The University of Tokyo, Yayoi 1-1-1, Bunkyo-ku, Tokyo 113-8657, Japan

$\$$ Department of Environmental Engineering, School of Civil and Environmental Engineering, University of Science and Technology Beijing, 30 Xueyuan Road, Haidian District, Beijing 10083, China

${ }^{\S}$ Beijing Key Laboratory on Resource-oriented Treatment of Industrial Pollutants, University of Science and Technology Beijing, 30 Xueyuan Road, Haidian District, Beijing 10083, China

"College of Bioresource Sciences, Nihon University, Kameino 1866, Fujisawa, Kanagawa 252-0880, Japan

${ }^{\perp}$ Division of Applied Physics, Faculty of Engineering Hokkaido University, N13W8 Kita-ku, Sapporo, Hokkaido 060-8628, Japan

* Corresponding author:

Seiichi Oshita, E-mail: aoshita@mail.ecc.u-tokyo.ac.jp ;

Shu Liu, E-mail: liushu0313055@gmail.com

TEL: 03-5841-5362

FAX: 03-5841-8174

\title{
Abbreviations list
}

Nanobubbles, NBs; Micro- and nano-bubbles, MNBs; Microbubbles, MBs; Dissolved oxygen, DO; Reactive oxygen species, ROS; 3'-p-(aminophenyl) fluorescein, APF; nitro blue tetrazolium, NBT

Number of figures and tables: 6 figures 0 tables Number of pages: 7 pages 


\section{NBT staining}

5 seeds in each group NBT: $1 \mathrm{mM}$

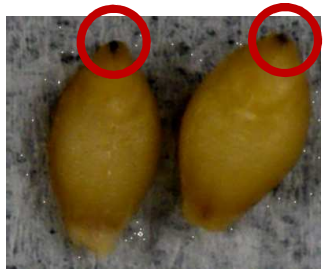

\section{Cutting process}

Frozen cube samples
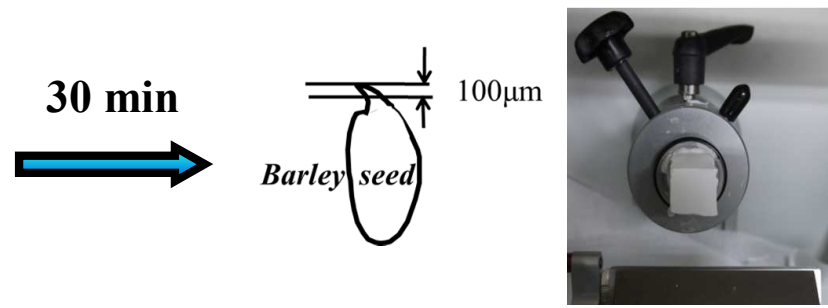

\section{ROS measurement}

Adhesive film

Section area: $0.1 \mathrm{~mm}^{2}$ (1st sliced)

$0.25 \mathrm{~mm}^{2}$ (2nd sliced)

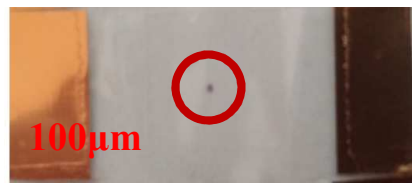

Microscope spectrophotometer

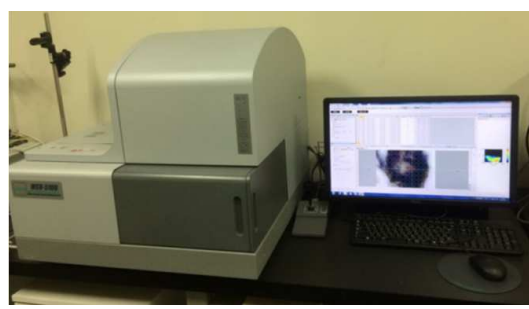

Figure S1. Schematic Diagram for the measurement of superoxide radical in seeds (The seeds in the photo were submerged in the water for 17 hours) 


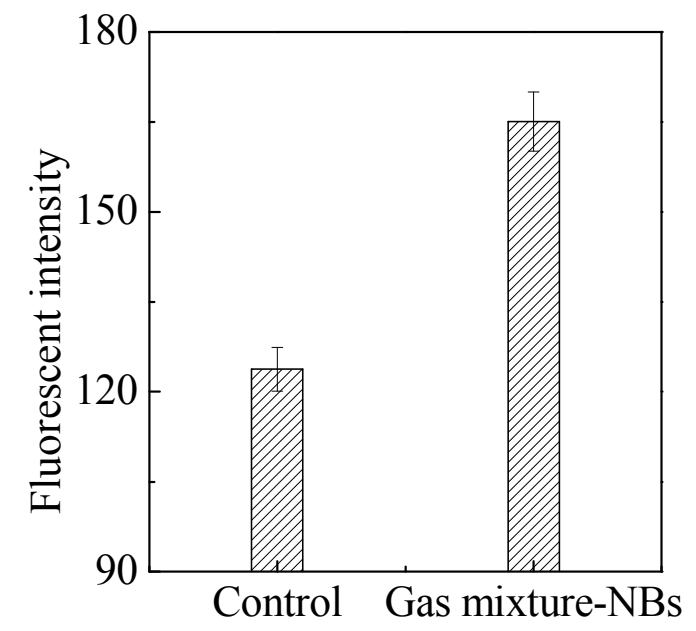

Figure S2. Fluorescence responses of $1 \mu \mathrm{M}$ APF to gas mixture NB water; Control was phosphate buffer without bubbles whose dissolved oxygen (DO) concentration was about $8.79 \mathrm{mg} \mathrm{L}^{-1}$. Gas mixture-NBs were generated for $1 \mathrm{~h}$ to obtain sufficient bubble numbers. DO concentrations of gas mixture NB water was $9 \mathrm{mg} \mathrm{L}^{-1}$. APF concentration was $1 \mu \mathrm{M}$. The photomultiplier voltage was $800 \mathrm{~V}$. 

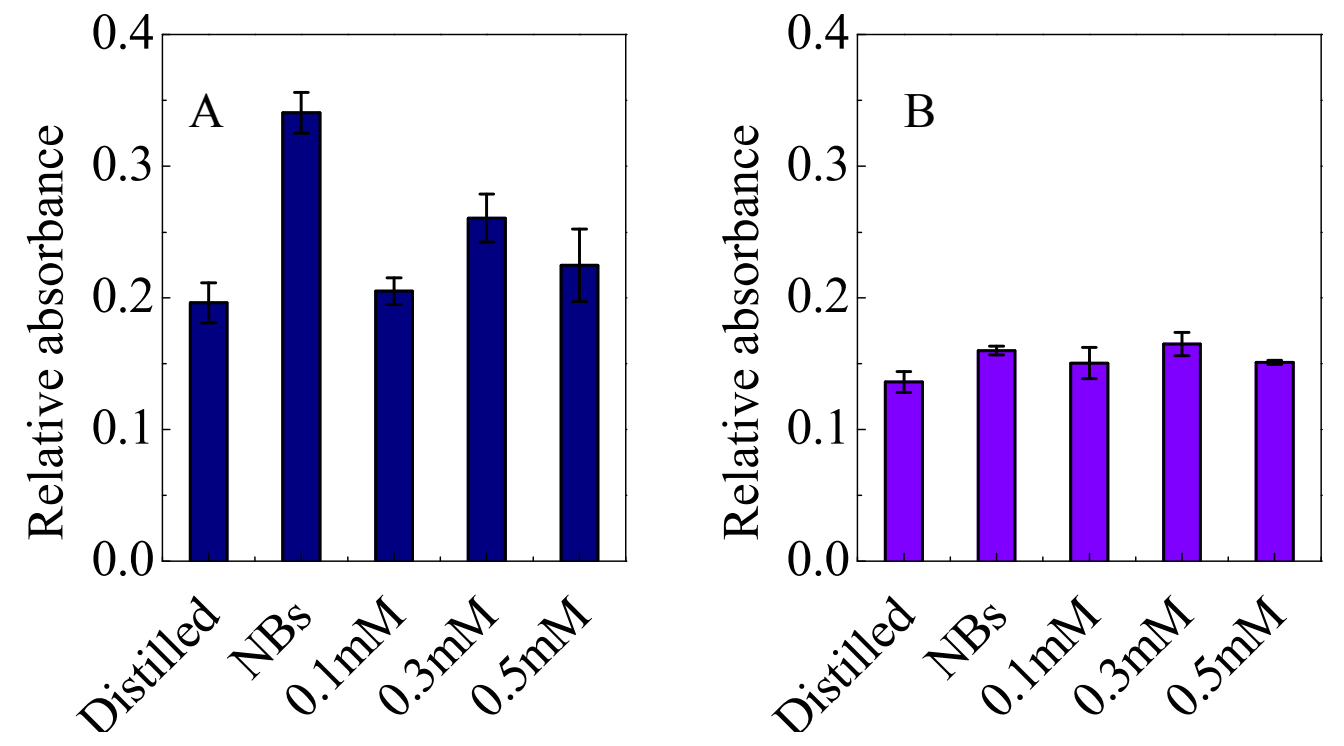

Figure S3 The average relative absorbance values of endogenous ROS generation in barley seeds germinated in five different solutions (Distilled water, NBs water, $0.1 \mathrm{mM}, 0.3 \mathrm{mM}$ and $0.5 \mathrm{mM} \mathrm{H}_{2} \mathrm{O}_{2}$ solutions). The Error bars show the standard error of five seeds in each group. A: dipping time $17 \mathrm{~h}$, B: dipping time $37 \mathrm{~h}$. 

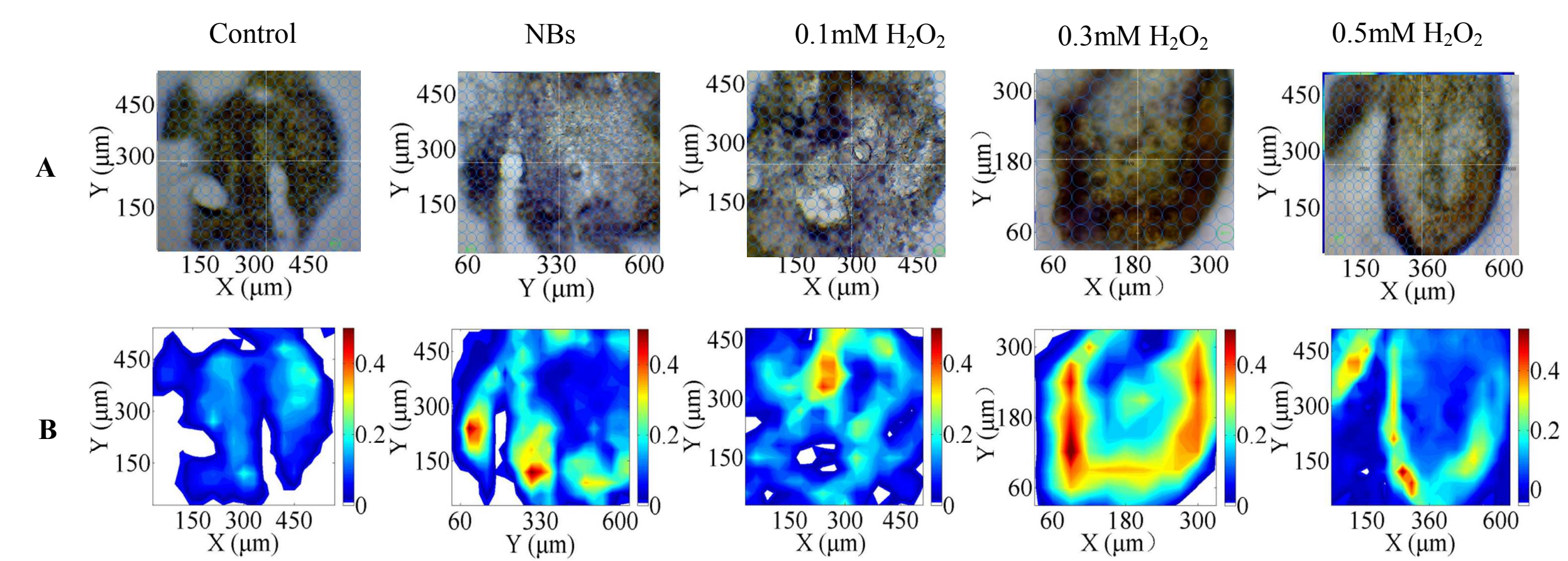

Figure S4. The distribution of endogenous reactive oxygen species (ROS) generation in barley seeds after $37 \mathrm{~h}$ of submerging time: the sprouting region of representative seeds germinated in five groups (distilled water, nanobubbles water, $0.1 \mathrm{mM}, 0.3 \mathrm{mM}$, and $0.5 \mathrm{mM} \mathrm{H}_{2} \mathrm{O}_{2}$ ) after the nitro blue tetrazolium staining process. (A): microscopic images of the sectioned surfaces of seeds germinated in five different kinds of water. Spectra were conducted on each pre-set circle. The diameter of each circle is $30 \mu \mathrm{m}$. (B): Superoxide radical distribution in samples. The $x$ - and $y$-axes show the locations of the measurement cycles. The measured values are the differences between absorbance values at $560 \mathrm{~nm}$ and $700 \mathrm{~nm}$ in each cycle area. 


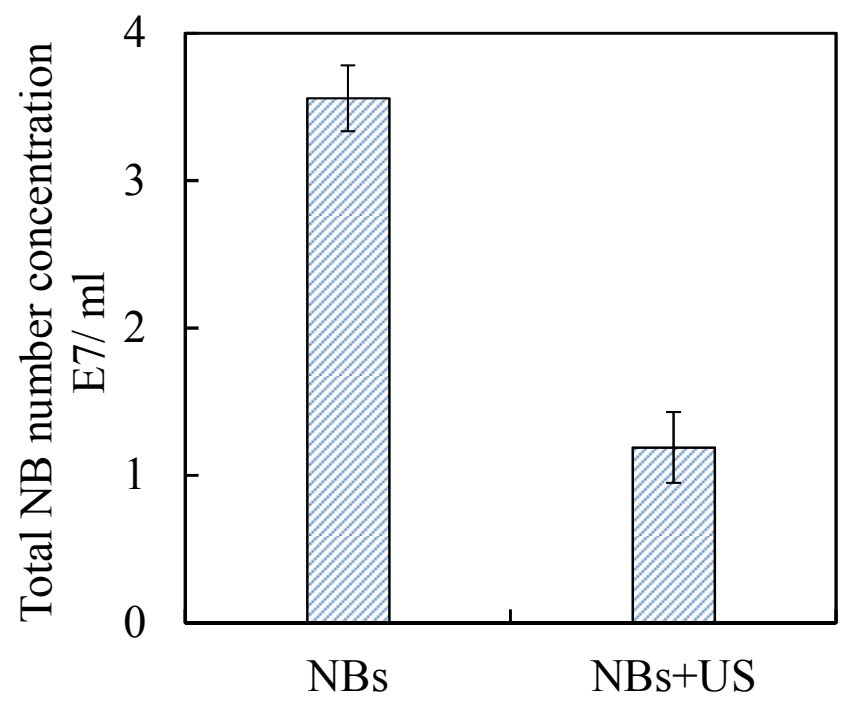

Figure S5. The effect of ultrasonic wave (US) on the total bubble number concentration in gas mixture NB water; The error bars show the standard errors of 3 measurements. 


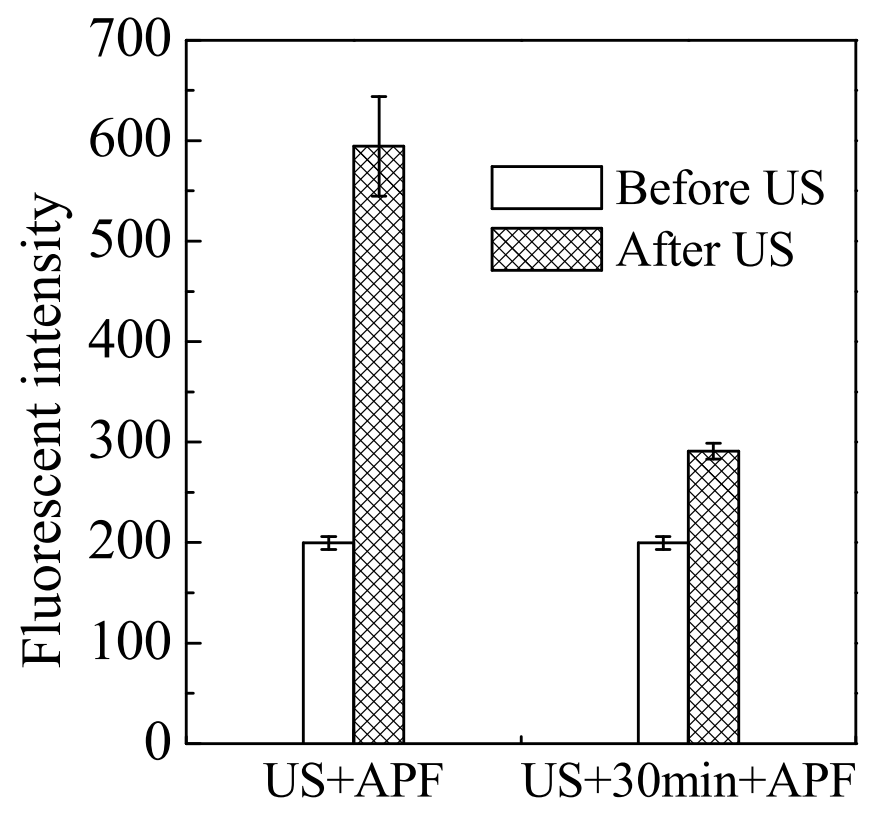

Figure S6. The effect of ultrasonic wave (US) on the fluorescent intensities of the buffer containing oxygen NBs (The values represent the fluorescent intensities at $515 \mathrm{~nm}$ with excitation at $490 \mathrm{~nm}$. The error bars show the standard errors of 8 parallel samples. US+APF: after the samples were taken out from the US device, APF was added; US+30min+APF: having taken out the sample from the US device, we wait for 30 minutes and then added APF. APF concentration was $1 \mu \mathrm{M}$ ) 